\title{
TU/e EmonOWEN

\section{Gels and glasses in a single system: Evidence for an intricate free-energy landscape of glassy materials}

\section{Citation for published version (APA):}

Jabbari-Farouji, S., Wegdam, G. H., \& Bonn, D. (2007). Gels and glasses in a single system: Evidence for an intricate free-energy landscape of glassy materials. Physical Review Letters, 99(6), 065701-1/4. [065701]. https://doi.org/10.1103/PhysRevLett.99.065701

DOI:

10.1103/PhysRevLett.99.065701

Document status and date:

Published: 01/01/2007

\section{Document Version:}

Publisher's PDF, also known as Version of Record (includes final page, issue and volume numbers)

\section{Please check the document version of this publication:}

- A submitted manuscript is the version of the article upon submission and before peer-review. There can be important differences between the submitted version and the official published version of record. People interested in the research are advised to contact the author for the final version of the publication, or visit the $\mathrm{DOI}$ to the publisher's website.

- The final author version and the galley proof are versions of the publication after peer review.

- The final published version features the final layout of the paper including the volume, issue and page numbers.

Link to publication

\section{General rights}

Copyright and moral rights for the publications made accessible in the public portal are retained by the authors and/or other copyright owners and it is a condition of accessing publications that users recognise and abide by the legal requirements associated with these rights.

- Users may download and print one copy of any publication from the public portal for the purpose of private study or research.

- You may not further distribute the material or use it for any profit-making activity or commercial gain

- You may freely distribute the URL identifying the publication in the public portal.

If the publication is distributed under the terms of Article 25fa of the Dutch Copyright Act, indicated by the "Taverne" license above, please follow below link for the End User Agreement:

www.tue.nl/taverne

Take down policy

If you believe that this document breaches copyright please contact us at:

openaccess@tue.nl

providing details and we will investigate your claim. 


\title{
Gels and Glasses in a Single System: Evidence for an Intricate Free-Energy Landscape of Glassy Materials
}

\author{
Sara Jabbari-Farouji, ${ }^{1}$ Gerard H. Wegdam, ${ }^{1}$ and Daniel Bonn ${ }^{1,2}$ \\ ${ }^{1}$ Van der Waals-Zeeman Institute, University of Amsterdam, 1018XE Amsterdam, the Netherlands \\ ${ }^{2}$ Laboratoire de Physique Statistique de l'ENS, 75231 Paris Cedex 05, France
}

(Received 28 November 2006; published 9 August 2007)

\begin{abstract}
In the free-energy landscape picture of glassy systems, their slow dynamics is due to a complicated free-energy landscape with many local minima. We show that for a colloidal glassy material multiple paths can be taken through the free-energy landscape. The evolution of the nonergodicity parameter shows two distinct master curves that we identify as gels and glasses. We show that for a range of colloid concentrations, the transition to nonergodicity can occur in either direction (gel or glass), accompanied by "hesitations" between the two. Thus, colloidal gels and glasses are merely global free-energy minima in the same free-energy landscape, and the paths leading to these minima can be complicated.
\end{abstract}

DOI: 10.1103/PhysRevLett.99.065701

The main issue in the study of glasses is that from the point of view of their molecular structure, they closely resemble liquids. On the other hand, their mechanical properties are much closer to those of solids: ordinary window glass, for instance, does not flow on human time scales [1]. To explain the extremely high viscosity of glassy systems, it is commonly accepted that the motion of molecules or particles that constitute the glass are blocked by the neighboring molecules, which in turn are blocked also by their neighbors and so on, making it impossible for the system to flow.

Translated in terms of the free energy of the system, the paradigm for glasses is that of a complicated free-energy landscape, with many local minima of the free energy $[2,3]$. The blocking of molecules or particles is then equivalent to saying that they are trapped in a local minimum of the free energy from which they cannot escape. This is most evident in the "aging" of such systems $[2,3]$ : just after a quench into the glassy phase, the system still evolves in the sense that the mechanical properties and the diffusion coefficient change in time. The interpretation of this time evolution is that at early times after its formation, the system is able to access at least part of the phase space, and can get out of local minima by thermal activation. However, as time goes on, the system finds deeper and deeper minima, more difficult to escape from, and consequently the evolution becomes slower. Because of this, the system cannot reach thermodynamic equilibrium: it becomes nonergodic. During this aging, the viscosity increases and the diffusion coefficient of the particles decreases, emphasizing the link between the blocking of the particle motion and absence of flow.

However, although this provides an appealing intuitive picture of glassy dynamics, to our knowledge there is no direct experimental evidence for the existence of such a complicated free-energy landscape with many local minima [4]. In this Letter we are able to provide such evidence by showing that for a soft glassy material multiple paths can be taken through the free-energy landscape that can
PACS numbers: 64.70.Pf, 81.40.Cd

even lead to different "final" nonergodic states at the late stages of aging.

The system we consider is a suspension of anisotropic and charged colloidal particles suspended in water: Laponite, a synthetic clay [5-10]. The study of colloids allowed for a significant contribution to elucidating the basic physics of glass transition $[11,12]$. In colloidal systems, as the particle volume fraction is increased, the particles become increasingly slower and for even higher volume fractions the glass transition is encountered. On the other hand, colloidal gels are known to form at extremely low volume fractions $10^{-4}-10^{-2}$ in the presence of strong attractions [13]. Gelation and the glass transition have important similarities. Both are ergodic to nonergodic transitions that are kinetic, rather than thermodynamic in origin, and distinguishing between these two types of nonergodic states experimentally is a long-standing controversy [14]. The experiments reported below provide direct criteria for distinguishing gels from glasses. This allows us to show that for a range of Laponite concentrations, two distinctly different nonergodic states can result at late times: either the glass or the gel forms at late times with roughly equal probability. There is no way to tell beforehand which of the two options will be taken by the sample, suggesting that there are at least two metastable minima in the system. In addition, our results show that the free-energy landscape is indeed complicated, since a number of samples are observed to hesitate between the two options for a long time and an initial evolution in one of the two directions can lead to a final state that is the other one.

The formation of nonergodic states in our system is followed in time using light scattering. For the experiments, we prepare Laponite XLG suspensions in ultrapure water. After stirring for $2 \mathrm{~h}$, we filtered to obtain a reproducible initial state [6]. This instant defines the zero of aging time, $t_{a}=0$. A standard dynamic light scattering setup measures the time-averaged intensity correlation functions $g_{t}(q, t)=\langle I(q, t) I(q, 0)\rangle_{t} /\langle I(q, 0)\rangle_{t}^{2}$, at scattering wave vector $q=(4 \pi n / \lambda) \sin \left(\frac{\theta}{2}\right)$, in which $\theta=90^{\circ}$ is the 
scattering angle. The aging time for which the timeaveraged correlation functions are not equal to their ensemble-averaged values, i.e., their values change from one position to another in the sample, defines the ergodicity-breaking point $t_{\mathrm{eb}}$. For $t_{a}>t_{\mathrm{eb}}$, we calculate the intermediate scattering function $f(q, t)$ from the timeaveraged intensity correlation function $g_{t}(q, t)$ and ensemble-averaged intensity $I_{E}$ measured by rotating the sample at different heights [11].

$$
f(q, t)=1+\left(I_{t} / I_{E}\right)\left\{\left[g_{t}(q, t)-g_{t}(q, 0)+1\right]^{1 / 2}-1\right\} .
$$

Figure 1 shows the intermediate scattering functions $f(q, t)$ for two different samples. Although there is a large difference in the typical waiting times (days for the lowconcentration sample and minutes for the highconcentration sample), the correlation functions evolve from an ergodic state to a nonergodic state: they no longer decay to zero for late times. However, low- and highconcentration samples are seen to behave in a distinctly different manner.

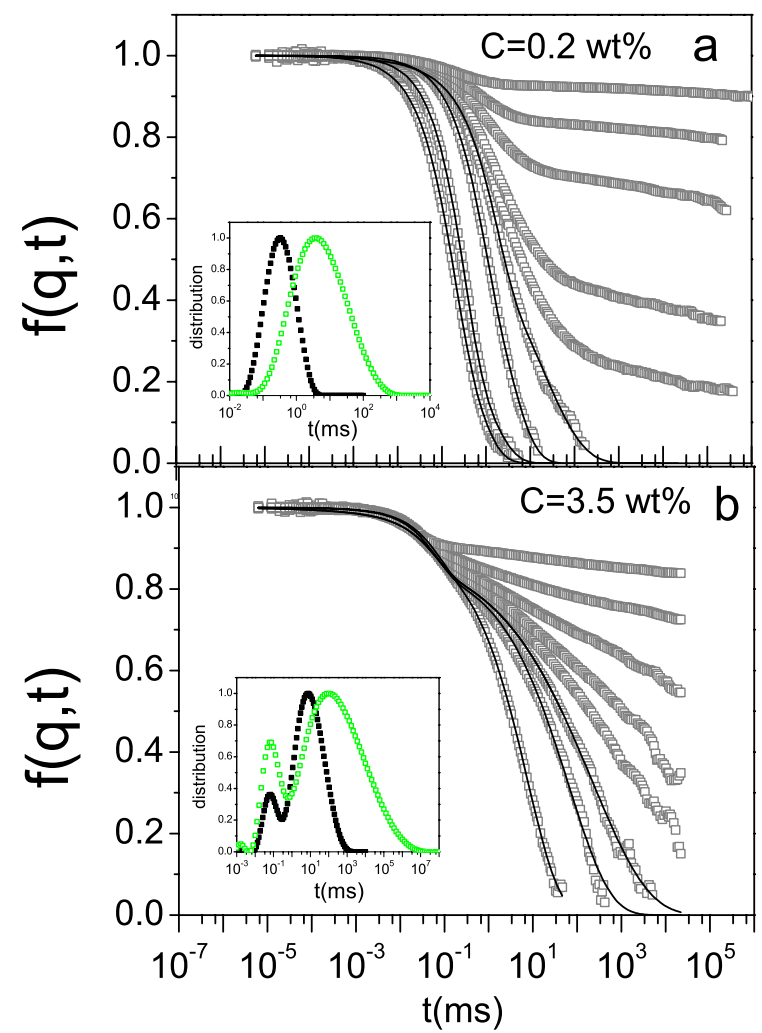

FIG. 1 (color online). Intermediate scattering function for increasing waiting times (from left to right) $t_{a}=1,49,71,80,85$, $89,91,99,112,141$ days for $C=0.2 \mathrm{wt} \%$ (a) and $t_{a}=7,40$, $54,71,86,113,260,1356 \mathrm{~min}$ for $C=3.5 \mathrm{wt} \%$ (b). The lines are fits. The insets show the distribution of relaxation times obtained directly from the correlation functions by a constrained regularization method (ALV-NonLin software data analysis) at an early stage of aging and just before ergodicity breaking $\left(t_{a}=\right.$ 7 and 80 days for $C=0.2 \mathrm{wt} \%$ and $t_{a}=7$ and $54 \min$ for $C=$ $3.5 \mathrm{wt} \%)$.
In the nonergodic regime, the aging rate of the system can be quantified by measuring the time evolution of the nonergodicity parameter $f\left(q, \infty, t_{a}\right)=\lim f\left(q, t \rightarrow \infty, t_{a}\right)$ [11]. Here, the long time limit $t \rightarrow \infty$ is taken with respect to the Brownian time scale $\tau_{B}=(2 R)^{2} / 6 D_{0} \approx 10^{-5} \mathrm{~s}$, i.e., $t \gg \tau_{B}$. We find that the evolution of the nonergodicity parameter collapses onto two distinct master curves when plotted as a function of reduced aging time $\left(t_{a} / t_{\mathrm{eb}}-1\right)$ [Fig. 2(a)] [15]. In the first group of samples (low concentrations) the nonergodicity parameter almost reaches unity: the colloidal particles are completely blocked, suggesting that they are rigidly held in place, as they would be in a gellike structure. In the second group (high concentrations), the nonergodicity parameter evolves at a slower rate and goes to $\approx 0.8$ at late times, indicating that there is still some freedom for the particles to move: the hindrance of the particles is only sterical, as it would be in a glass. Measuring the nonergodicity parameter for a couple of glassy samples until $t_{a} / t_{\mathrm{eb}}=15$, we found that $f(q, \infty)$ never exceeds 0.835 .

Perhaps the most striking observation is that for intermediate concentrations $1.3<C<2.3 \mathrm{wt} \%$, identical samples may evolve at very different rates, thus having a very different ergodicity-breaking point. Figure 2(b), shows the ergodicity-breaking time of the ensemble of the samples we have measured as a function of concentra-
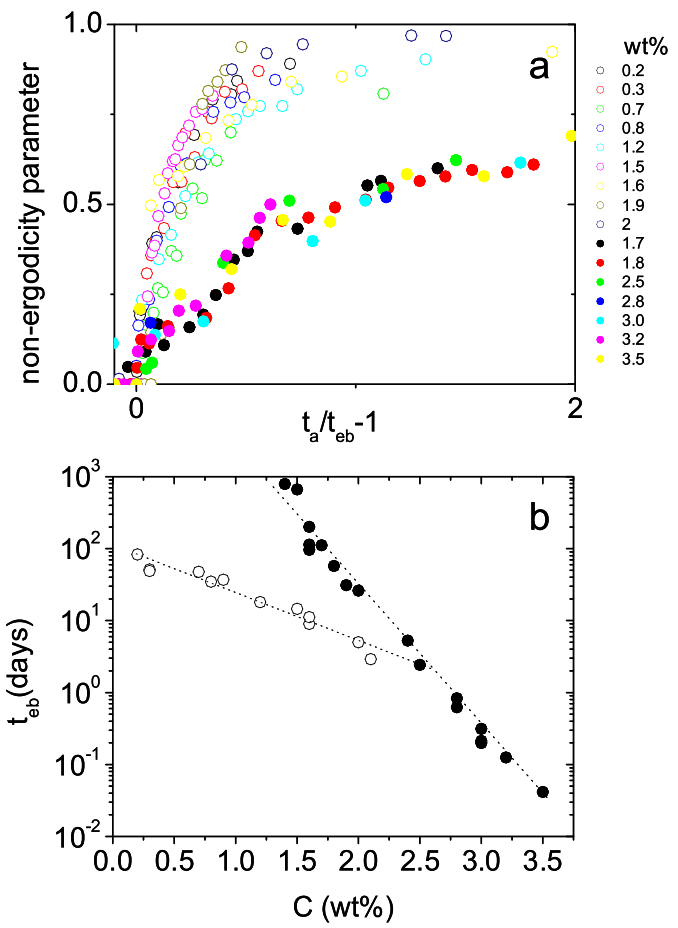

FIG. 2 (color online). (a) Evolution of the nonergodicity parameter $f(q, \infty)$ for different samples. The samples can be divided into two groups according to the evolution of nonergodicity parameters. In both figures (and the following ones), the open symbols correspond to gels, the filled symbols correspond to the glass. (b) The ergodicity-breaking time as a function of concentration. 
tion. The samples fall in two separate groups, and the samples in the intermediate concentration region fall in either of the two. This suggests that the intermediate concentration samples have two options, either following the same trend as the samples of high concentration or behaving similarly to the samples of low concentrations.

We would like to emphasize that these results are not dependent on the sample preparation. To investigate the effect of sample preparation, we prepared a large number of Laponite samples (of the order of 100) with different concentrations in large amounts. Part of each sample was placed in a cuvette for light scattering experiments and part of it was kept in a bottle. The samples in the cuvettes and bottles prepared in exactly the same way could either age at the same rate or differently; i.e., the sample in the cuvette could become solidlike while the one in bottle remained liquid or vice versa.

The difference between the two states becomes clear if we look at the rest of the data. Before ergodicity breaking, as can be observed from the fits shown in Fig. 1, both the low- and the high-concentration samples can be described by a sum of a single exponential and stretched exponential $A \exp \left(-t / \tau_{1}\right)+(1-A) \exp \left[-\left(t / \tau_{2}\right)^{\beta}\right][7,8] . \tau_{1}$ is related to the inverse of the short-time diffusion $\tau_{1}=1 /\left(D_{s} q^{2}\right)$ (Fig. 3). $D_{s}$ is almost a constant for the samples of high concentration $(C>2.3 \mathrm{wt} \%)$; this corresponds to the "rattling in the cage" motion reported earlier for colloidal glasses [7]. However, $D_{s}$ decreases significantly with aging time for low concentrations $(C<1.4 \mathrm{wt} \%)$ which is again related to the incorporation of the colloidal particles in a gel network. In addition, the slow relaxation time $\tau_{2}$ grows exponentially with aging time for high concentrations, in agreement with earlier observations for the glassy state $[7,8]$. However, for the gel phase, the relaxation time increases faster than exponentially; this is likely to be related to the formation of small clusters in the beginning that subsequently aggregate to form a macroscopic structure (with a large relaxation time) [8,9]. To disentangle the effect of short-time from long-time relaxation, in Fig. 4(a) we have plotted the ratio $\tau_{2} / \tau_{1}$.

The measured static structure factor Fig. 5 provides two further pieces of evidence for structure formation. First, for

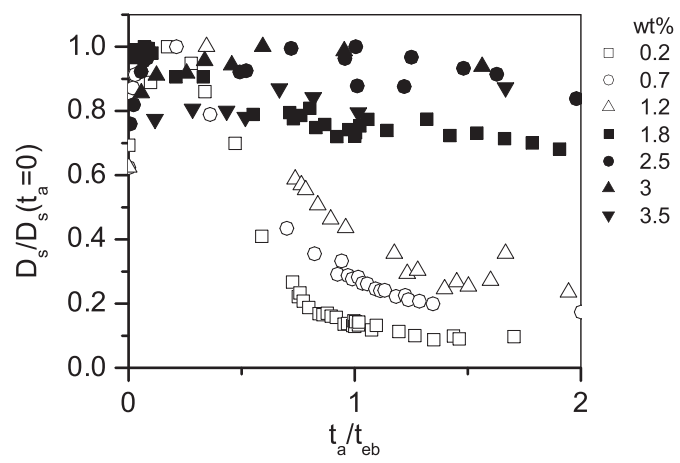

FIG. 3. The evolution of short-time translational diffusion normalized to its initial value $\left(t_{a} \approx 0\right)$ as a function of $t_{a} / t_{\mathrm{eb}}$. all the low-concentration samples, the scattered intensity consistently increases with aging time, while for the highconcentration ones it does not evolve much; see Fig. 5(a). The increase of scattered light intensity is usually attributed to formation of a network or clusters of particles [13]. Second, although the range of wave vectors is rather limited, measurement of the static structure factor $S(q)$ for the different samples, a power law behavior for $S(q)$ appears to be observed for the lowest concentration samples with an exponent $1.1 \pm 0.2$, a characteristic of a stringlike cluster. To the contrary, an almost flat structure factor is found for the highest concentrations, indicating homogeneity, Fig. 5(b), very characteristic of a glass [6]. The noise at low $q$ for these measurements is probably due to imperfections of the measurement cell that scatter light at small angles.

Combining all these data, we identify the lowconcentration samples as colloidal gels and the highconcentration ones as colloidal glasses. Intermediate concentrations can be either gels or glasses at late times, with no way of telling beforehand how the sample is going to evolve. That the path towards these nonergodic states is complicated follows from the observation that samples may "hesitate" for a long time between the two states, and may evolve in one direction to end up in the other. In Fig. 4 some of the "hesitating" samples are shown. It clearly shows how a few samples in the intermediate concentration region that behaved consistently like glassy samples before the ergodicity-breaking point end up as gels at late times. Perhaps even more surprisingly, Fig. 4(b) shows that even if one looks at a single observable such as the nonergodicity parameter, a crossover between the two behaviors can be observed; this is most evident in the data for $1.9 \mathrm{wt} \%$. The data shown in the figure are mere examples; approximately $20 \%$ of the samples in the intermediate concentration region behaved in an ambiguous way in the sense that they seemed to have a hard time to "decide" whether they were glasses or gels.

The nature of the nonergodic state in Laponite suspensions has been the subject of considerable controversy: both colloidal gel [5,9] and colloidal glass formation

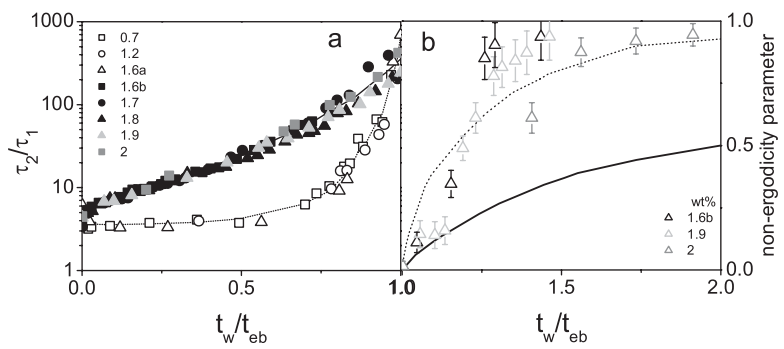

FIG. 4. (a) The ratio $\tau_{2} / \tau_{1}$ and (b) nonergodicity parameter as a function of scaled aging time $t_{a} / t_{\mathrm{eb}}$. These samples behave like a glass at the early stages of aging and after $t_{a} / t_{\mathrm{eb}} \approx 1.2$ they evolve as gels. The full lines show the glassy and dotted lines show the gel line, obtained by averaging over all the samples measured. 


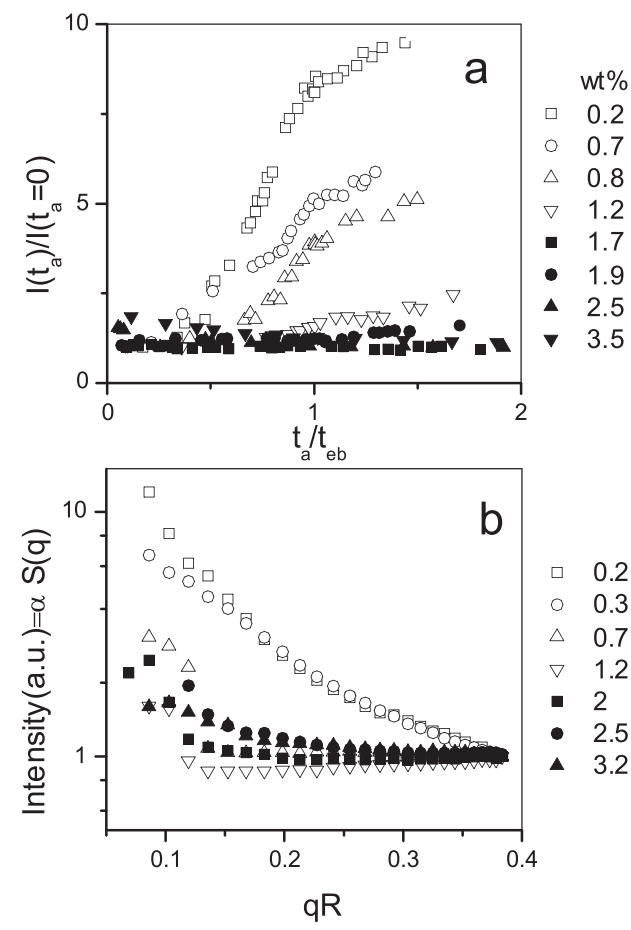

FIG. 5. (a) Scattered intensity as a function of aging time. We have normalized the intensity to its value at the beginning of aging. (b) The scattered intensity as a function of dimensionless wave vector $q R$ for several Laponite concentrations. To compare the $q$ dependence of different samples, we normalized the data to their value at highest measured $q R=0.38$.

$[6,10]$ have been invoked and were thought to be mutually exclusive $[6,9]$. We here provided clear experimental evidence for how the distinction of gel and glassy states can be made [16]. This allows for the observation that for intermediate concentrations, the transition to nonergodicity can occur in either direction (gel or glass), and may be accompanied by "hesitations" between the two directions. A qualitative explanation for this behavior is provided by the free-energy landscape picture.

For Laponite our data suggest that there are at least two metastable minima in the free energy corresponding to gel and glass states, and that different pathways towards these nonequilibrium states exist. Further evidence comes from the distribution of relaxation times obtained from the correlation functions of gel-like and glassy samples, assuming that the correlation function is a superposition of exponential modes $f(q, t)=\int_{\tau_{\min }}^{\tau_{\max }} \exp (-t / \tau) G(\tau) d \tau$. As can be seen from the insets in Fig. 1, the distribution function of relaxation times has a completely different form for gels and glassy samples. The gels show a broad spectrum of relaxation times, whereas for the glasses there is a distinction between short- and long-time diffusive behavior. With increasing aging time, the distributions become wider and the average relaxation time grows for both types of samples. The relaxation time of the system is usually assumed to be proportional to exponential of the corresponding energy barrier [17]. Therefore, the different dis- tribution of relaxation times corresponds to different distribution of energy barriers for gels and glasses, thus providing another piece of evidence for the existence of a complicated free-energy landscape.

This should be general for colloidal systems with attractive interactions between the particles; indeed the recent discovery of "attractive glasses" for spherical colloids [12] also suggests that gels and glasses are not necessarily clearly distinct states of matter, but rather metastable minima in an otherwise complicated free-energy landscape, resulting from both steric and attractive interactions, as also suggested by some simulations [18].

[1] E. D. Zanottoa, Am. J. Phys. 66, 392 (1998).

[2] J. P. Bouchaud, J. Phys. I (France) 2, 1705 (1992).

[3] S. Sastry et al., Nature (London) 393, 554 (1998); S. Sastry, et al., Physica (Amsterdam) 270A, 301 (1999); F. H. Stillinger and P. G. Debenedetti, J. Chem. Phys. 116, 3353 (2002).

[4] L. Leuzzi and T.M. Niewenhuizen, Phys. Rev. E 64, 066125 (2001); C. Donati et al., Phys. Rev. Lett. 85, 1464 (2000).

[5] A. Mourchid et al., Langmuir 11, 1942 (1995); P. Levitz et al. Europhys. Lett. 49, 672 (2000).

[6] D. Bonn et al., Langmuir 15, 7534 (1999); Europhys. Lett. 45, 52 (1999).

[7] B. Abou et al., Phys. Rev. E 64, 021510 (2001).

[8] B. Ruzicka et al., Phys. Rev. Lett. 93, 258301 (2004); Langmuir 22, 1106 (2006).

[9] P. Mongondry et al., J. Colloid Interface Sci. 283, 397 (2005); T. Nicolai and S. Cocard, J. Colloid Interface Sci. 244, 51 (2001).

[10] M. Bellour et al., Phys. Rev. E 67, 031405 (2003); S. Kaloun et al. Phys. Rev. E 72, 011403 (2005).

[11] P. N. Pusey and W. van Megen, Phys. Rev. Lett. 59, 2083 (1987); W. van Megen et al., Phys. Rev. Lett. 67, 1586 (1991).

[12] K. N. Pham et al., Science 296, 104 (2002); K. N. Pham et al., Phys. Rev. E 69, 011503 (2004); K. Kroy et al., Phys. Rev. Lett. 92, 148302 (2004).

[13] D. A. Weitz et al., Phys. Rev. Lett. 54, 1416 (1985); M. Carpineti and M. Giglio, Phys. Rev. Lett. 68, 3327 (1992); P. N. Segré et al. Phys. Rev. Lett. 86, 6042 (2001).

[14] H. Tanaka et al., Phys. Rev. E 69, 031404 (2004); H. Tanaka et al., Phys. Rev. E 71, 021402 (2005).

[15] Note that the nonergodicity parameter grows continuously from zero to higher values for both groups of samples. Because of our way of defining it, this differs from the usual jump from zero to a finite value that generally occurs when taking the infinite aging time limit $t_{a} \rightarrow \infty$.

[16] At the concentration where gel forms this was previously thought to be fluid phase [5] or a Wigner glass composed of Laponite clusters [8]. Also we consider the highconcentration samples to be merely a glass phase and not a Wigner glass as was suggested before [6].

[17] P. A. Egelstaff, An Introduction to the Liquid State (Academic, New York, 1967).

[18] F. Sciortino et al., Phys. Rev. Lett. 93, 055701 (2004). 